Article

\title{
Use of Repetitive Sequences for Molecular and Cytogenetic Characterization of Avena Species from Portugal
}

\author{
Diana Tomás ${ }^{1}$, Joana Rodrigues ${ }^{1}$, Ana Varela ${ }^{1}$, Maria Manuela Veloso ${ }^{1,2}$, Wanda Viegas ${ }^{1}$ and \\ Manuela Silva ${ }^{1, *}$ \\ 1 Linking Landscape, Environment, Agriculture and Food (LEAF), Instituto Superior de Agronomia, \\ Universidade de Lisboa, Tapada da Ajuda, 1349-017 Lisboa, Portugal; dianarstomas@isa.ulisboa.pt (D.T.); \\ joana.jr.rodrigues@gmail.com (J.R.); amsvg_89@hotmail.com (A.V.); mveloso.inrb@gmail.com (M.M.V.); \\ wandaviegas@isa.ulisboa.pt (W.V.) \\ 2 Instituto Nacional de Investigação Agrária e Veterinária, Quinta do Marquês, 2784-505 Oeiras, Portugal \\ * Correspondence: manuelasilva@isa.ulisboa.pt; Tel.: +351-213-653-281; Fax: +351-213-653-238
}

Academic Editor: Jianhua Zhu

Received: 5 November 2015; Accepted: 26 January 2016; Published: 4 February 2016

\begin{abstract}
Genomic diversity of Portuguese accessions of Avena species-diploid A. strigosa and hexaploids $A$. sativa and A. sterilis-was evaluated through molecular and cytological analysis of $45 \mathrm{~S}$ rDNA, and other repetitive sequences previously studied in cereal species-rye subtelomeric sequence (pSc200) and cereal centromeric sequence (CCS1). Additionally, retrotransposons and microsatellites targeting methodologies-IRAP (inter-retrotransposon amplified polymorphism) and REMAP (retrotransposon-microsatellite amplified polymorphism)-were performed. A very high homology was detected for ribosomal internal transcribed sequences (ITS1 and ITS2) between the species analyzed, although nucleolar organizing regions (NOR) fluorescent in situ hybridization (FISH) analysis revealed distinct number of Nor loci between diploid and hexaploid species. Moreover, morphological diversity, evidenced by FISH signals with different sizes, was observed between distinct accessions within each species. pSc200 sequences were for the first time isolated from Avena species but proven to be highly similar in all genotypes analyzed. The use of primers designed for CCS1 unraveled a sequence homologous to the Ty3/gypsy retrotransposon Cereba, that was mapped to centromeric regions of diploid and hexaploid species, being however restricted to the more related $\mathrm{A}$ and $\mathrm{D}$ haplomes. Retrotransposon-based methodologies disclosed species- and accessions-specific bands essential for the accurate discrimination of all genotypes studied. Centromeric, IRAP and REMAP profiles therefore allowed accurate assessment of inter and intraspecific variability, demonstrating the potential of these molecular markers on future oat breeding programs.
\end{abstract}

Keywords: Avena Portuguese lines; repetitive sequences; genomic diversity; molecular markers

\section{Introduction}

Avena genus $(2 n=2 x=14)$ comprises diploid (A and $\mathrm{C}$ genomes), tetraploid ( $\mathrm{AB}$ and $\mathrm{AC}$ genomes) and hexaploid (ACD) species. Hexaploid species appear to have arisen by two rounds of hybridization followed by chromosome duplication whereas tetraploid species with AB genomes were suggested to result from an unrelated event involving an A diploid species autopolyploidization (for a review see [1]). Structurally different $A$ and $C$ genomes have variants and $B$ and $D$ genomes are considered to be derived from an A genome [1]. Only four of 27 Avena species [2] are cultivated and used for food, feed or forage, namely: diploid A. strigosa (A), tetraploid A. abyssinica (AB) and hexaploids 
A. byzantine and A. sativa (ACD) [1]. A. strigosa and A. sativa are the major Avena crops in Portugal, which is considered the center of diversity of the former one [1]. On the other hand, the most relevant Avena wild species in Portugal is A. sterilis [3] with a high potential in oat breeding programs due to important agronomic traits increased grain yield and protein content and resistance to abiotic and biotic stresses [4]. In fact, natural hybrids between $A$. sativa and $A$. sterilis occur frequently and are widespread in Portugal [3].

Several molecular markers such as Restriction Fragment Length Polymorphism (RFLP), Amplified Fragment Length Polymorphism (AFLP), microsatellites and rDNA sequences were previously used to survey genomic diversity in Avena genus [5-7]. Avena genus phylogenetic correlations have been evaluated through the analysis of 45S rDNA Internal Transcribed Spacers (ITS1 and ITS2) of species with distinct genome composition [8-11]. On the other hand, RFLP, microsatellites and Inter-Simple Sequence Repeat (ISSR) were used to geographically cluster accessions of $A$. strigosa [12], A. sativa $[13,14]$ and $A$. sterilis $[15,16]$. Inter-retrotransposon amplified polymorphism (IRAP) and retrotransposon-microsatellite amplified polymorphism (REMAP) methodologies were initially designed to identify different barley (H. vulgare) cultivars [17]. Such PCR-based techniques target retrotransposons long terminal repeat (LTR) sequences relevant in genome evolution and speciation due to their mobile nature [18] and microsatellites loci that are preferentially associated with retrotransposons in cereals [19]. Those methodologies were recently used for genomic characterization of monocotyledonous crop species such as wheat (T. aestivum), rye (S. cereale), triticale (xTriticosecale) (reviewed in [20]), rice (O. sativa) [21], maize (Z. mays) [22], cocoyam (Xanthosoma sagittifolium) and taro (Colocasia esulenta) [23]. REMAP methodologies were also previously used as markers associated with A. sativa agronomic traits [24] as well as to assess $A$. sativa, A. sterilis and A. fatua evolutionary relationships [25]. Molecular characterization of such dispersed sequences as well as other repetitive sequences is therefore important to better evaluate Avena landraces genomic diversity. Rye subtelomeric pSc200 sequences for instance were already relevant to disclose differences between cereal genera such as Secale, Triticum and Hordeum [26] although not yet studied in Avena. A similar situation occurs with the highly conserved cereal centromeric sequence (CCS1) firstly isolated from Brachypodium sylvaticum [27] and already used to characterize several species as barley (Hordeum vulgare), wheat (T. aestivum), rye (S. cereale), maize (Zea mays) and rice (Oryza sativa) either through Southern blot, PCR analysis or fluorescent in situ hybridization [27,28].

In this work, the characterization of distinct Portuguese accessions of A. strigosa, A. sativa and A. sterilis was performed through the analysis of distinct repetitive genome fractions, including Nor loci (ITS1 and ITS2) as well as sequences previously mapped in other cereals at centromeric (CCS1) and subtelomeric (pSc200) chromosome domains. Sequences dispersed throughout the genome such as retrotransposons and microsatellites were also analyzed through IRAP and REMAP methodologies. Besides interspecific variability, the results obtained also disclosed intraspecific genetic diversity in Portuguese Avena species allowing the establishment of molecular markers that may be useful to reliably identify valuable genotypes.

\section{Results}

To assess Avena diversity we analyzed distinct repetitive sequences such as 45S rDNA ITS1 and ITS2 and sequences previously mapped on subtelomeric (pSc200) and centromeric (CCS1) domains in other cereal species. Retrotransposons and microsatellites flancking sequences were also analyzed through IRAP (Inter Retrotransposons Amplified Polymorphism) and REMAP (Retrotransposons Microsatellite Amplified Polymorphism) methodologies. All molecular markers used revealed unique and consistent banding profiles in at least three distinct individuals of each genotype of A. strigosa, A. sativa and A. sterilis demonstrating the inexistence of intravarietal variability. The total number of bands per molecular marker used and genotype analyzed are summarized in Table 1. $45 \mathrm{~S}$ rDNA ITS1 and ITS2 and pSc200 amplification yielded a single band in all Avena genotypes that was further isolated, purified and cloned for sequences analysis (Accession Numbers in Table S1). 
Centromeric sequences, IRAP and REMAP yielded distinct banding profiles for each genotype which were further compared using NTSYSpc software (Numerical Taxonomy and Multivariate Analysis System).

Table 1. Number of bands per banding profile obtained in each PCR experiment.

\begin{tabular}{ccccccc}
\hline Accession & 45S ITS1 & 45S ITS2 & pSc200 & CCS1 & IRAP & REMAP \\
\hline A. strigosa "Madeira Island" & 1 & 1 & 1 & 5 & 9 & 15 \\
A. strigosa "Elvas" & 1 & 1 & 1 & 5 & 9 & 14 \\
A. sativa "Kyto" & 1 & 1 & 1 & 6 & 10 & 14 \\
A. sativa "Madeira Island" & 1 & 1 & 1 & 7 & 8 & 9 \\
A. sativa "S. Eulália" & 1 & 1 & 1 & 5 & 11 & 13 \\
A. sterilis & 1 & 1 & 1 & 4 & 10 & 9 \\
\hline
\end{tabular}

ITS1 and ITS2: 45S rDNA internal transcribed spacers; pSc200: rye subtelomeric sequence (Accession Number Z50039); CCS1: cereal centromeric sequences (Accession Number, U52217), IRAP Nikita and REMAP Nikita (CA)9G.

\subsection{S rDNA Internal Transcribed Sequences Similarity and Nor Loci Diversity}

Primers targeting 45S rDNA sequences [29] produced single bands with the expected sizes ( 300 and $\sim 380 \mathrm{bp}$, respectively) in all species/accessions analyzed as well as in pTa71 plasmid used as control. ITS1 and ITS2 sequences included in those amplified products present 219 and $216 \mathrm{bp}$, respectively, as already described for other Avena species [11]. Moreover, ITS sequence analysis using original parameters of Clustal W2 [30] revealed a high level of similarity-between $98 \%$ and $100 \%$ for ITS1 and between $99 \%$ and $100 \%$ for ITS2. ITS1 sequences alignment presented six polymorphic nucleotides and $100 \%$ identity was detected between A. sativa "S. Eulália" and A. sterilis. In ITS2 sequences only one polymorphic nucleotide was detected between $A$. strigosa and A. sativa "Madeira Island" from A. sativa Kyto, S. Eulália and A. sterilis.

Nor loci evaluation performed in root tip cells of $A$. strigosa landraces revealed four FISH positive NORs using pTa71 probe (Figure 1), presenting however some intraspecific morphological variability. In fact, in "Madeira Island" landrace two pairs of NORs with distinct sizes are observed (Figure 2a) and in "Elvas" landrace the four NORs exhibit similar sizes (Figure 2b).
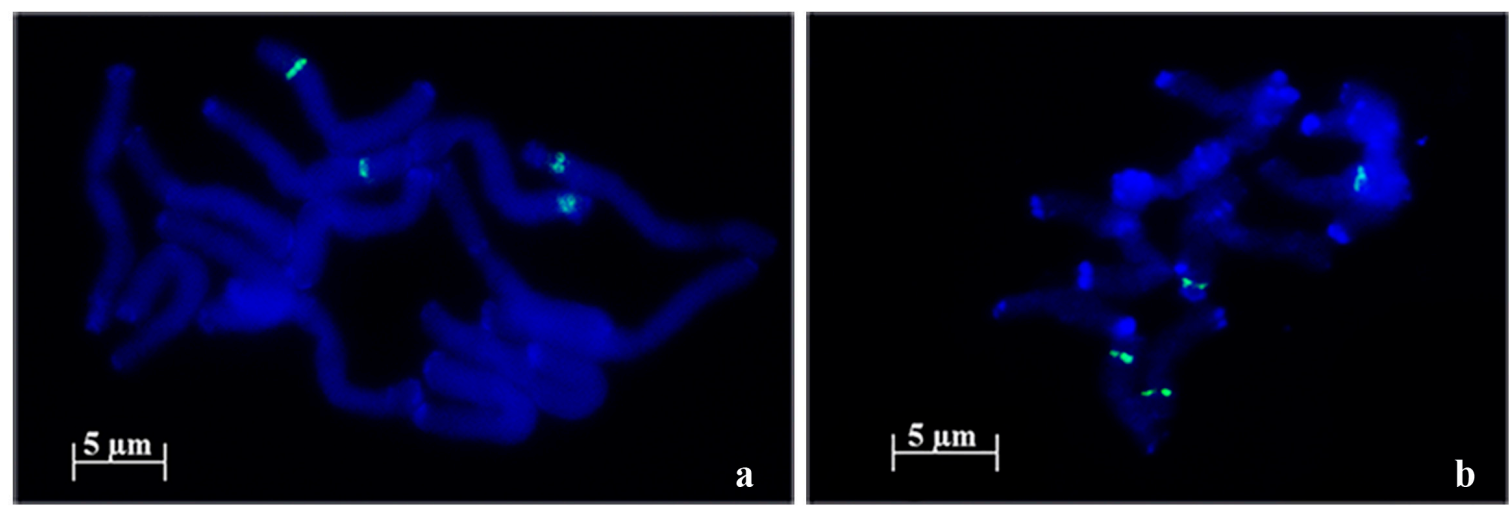

Figure 1. $45 \mathrm{~S}$ rDNA fluorescent in situ hybridization (green signals) in metaphase cells of diploid A. strigosa. In the landrace from Madeira Island (a) two pairs of nucleolar organizing regions (NORs) with distinct sizes are observed and in the landrace from Elvas; (b) two pairs of NORs with similar sizes are detected.

In hexaploid species $A$. sativa and $A$. sterilis, $45 \mathrm{~S}$ rDNA FISH revealed three pair of signals (Figure 2). However, in A. sativa distinct landraces revealed Nor loci diversity in relation to NORs size. While in "S. Eulália" all three NOR pairs exhibit similar sizes (Figure 2a), "Madeira Island" landrace 
presented FISH signals with different sizes corresponding to one pair of large NORs, one pair of medium size NORs and one heteromorphic pair composed by one medium size and one minor NOR (Figure 2b). Furthermore, in A. sterilis prometaphase cells with distended chromosomes, the three NOR pairs are distinguished through their different topological organization since one pair presents a single FISH domain whereas the other two pairs show more than one labeled domain intercalated by an unlabeled region that corresponds most probably to transcriptionally active uncondensed rDNA units. In one pair of NORs the two domains have similar dimensions, contrasting with the other pair where both domains have different dimensions (Figure 2c). The numbers of rDNA FISH signals and their described organization patterns were observed in at least 10 metaphase or prometaphase cells and are summarized in Table 2.
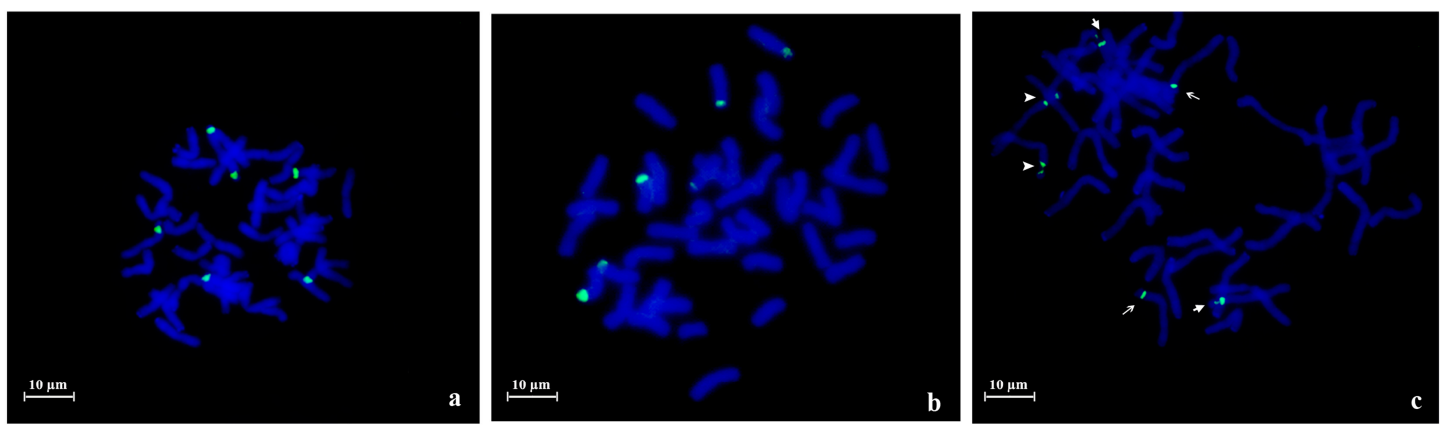

Figure 2. $45 \mathrm{~S}$ rDNA fluorescent in situ hybridization (green signals) in metaphase cells of Avena hexaploid species. Three pairs of NORs are detected both in A. sativa as in A. sterilis. (a) A. sativa “S. Eulália" presenting NORs with similar sizes; (b) A. sativa from Madeira Island showing one large pair of NORs, one medium size pair and one heteromorphic pair (one medium size and one small); (c) A. sterilis showing one NOR pair with one signal (long line arrow); a second pair composed by two domains with different sizes (short line arrow); and a third one with two domains with similar sizes (arrow head).

Table 2. rDNA FISH signals.

\begin{tabular}{cccc}
\hline Species & Accession & Number of NORs & Description \\
\hline \multirow{2}{*}{ A. strigosa } & "Madeira" & 4 & 1 large pair and 1 small pair \\
\cline { 2 - 4 } A. sativa & "Elvas" & 4 & 2 pairs with similar size \\
\cline { 2 - 4 } & "Madeira Island" & 6 & $\begin{array}{c}\text { 1 large pair, 1 medium pair and 1 } \\
\text { "S. Eulália" }\end{array}$ \\
\cline { 2 - 4 } A. sterilis & - & 6 & 3 pairs with similar sizes \\
\hline
\end{tabular}

\subsection{Novel Repetitive Sequences Were Identified Contributing for Avena Accessions Discrimination}

Genomic diversity evaluation in Avena was extended through the analysis of repetitive sequences previously described in subtelomeric and centromeric domains in other cereal species [26]. The amplification products obtained with primers designed to pSc200 sequence are presented in Figure 3. A $444 \mathrm{bp}$ fragment is obtained, as a result of the amplification between the forward and reverse primers in subsequent units, as already described in [31], including in all species/accessions analyzed a single complete unit with $379 \mathrm{bp}$ as described in rye [32], and where a significantly more intense band is observed when rye genomic DNA is used as control. Those bands were isolated, cloned and sequenced (Accession Numbers in Table S1) and their BLAST (Basic Local Alignment Search Tool) analysis in NCBI database revealed between $99 \%-100 \%$ similarity with rye subtelomeric sequence 
(Accession Number Z50039, [32] and 90\% homology with a A. sativa sequence (Genbank Accession Number DQ481575, unpublished data). Moreover, the homology level between pSc200-like sequences isolated from the distinct Avena genotypes analyzed (alignment in Figure S1) is extremely high ( 99\%).

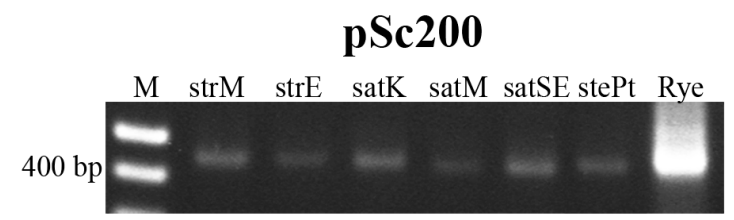

Figure 3. PCR amplification products obtained using pSc200 primers. M: molecular marker 1 $\mathrm{Kb}+$; strM-A. strigosa from Madeira Island; strE-A. strigosa from Elvas; satK-A. sativa "Kyto"; satM-A. sativa from Madeira Island; satSE-A. sativa "S. Eulália"; stePt—A. sterilis Pt; Rye—S. cereale.

FISH analysis on the Avena genotypes studied was performed using pSc200 probe and rDNA pTa71 probe as positive control. Contrary to the rDNA probe that was detected in all metaphase spreads, the pSc200 probe was not detected in any Avena accession.

Another repetitive sequence hitherto mapped in cereal centromeric regions (CCS1) was studied in Avena through PCR amplification and FISH evaluation. The PCR profiles obtained are distinct in the three Avena species analyzed and contain bands ranging in size from 350 to $2500 \mathrm{bp}$ (schematic representation in Figure 4). Two bands are common to all genotypes analyzed, one with a size identical (950 bp) to the published maize centromeric sequence [28] and a novel one with $700 \mathrm{bp}$. Both $A$. strigosa landraces exhibit identical profiles, containing besides the two common bands, three other bands, two A. strigosa-specific ( $\sim 380$ and $\sim 1700 \mathrm{bp}$ ) and one common to A. sativa ( $\sim 1650 \mathrm{bp})$. A. sativa and A. sterilis banding profiles present one band characteristic of Avena hexaploid species with $\sim 2300 \mathrm{bp}$ as well as a A. sativa-specific band with $\sim 2500 \mathrm{bp}$, which distinguishes the hexaploid species studied. Also, A. sativa landraces present very similar profiles composed of five $A$. sativa common bands previously described, plus "Kyto" or "Madeira Island" specific bands ( 900 and $~ 1100 \mathrm{bp}$, respectively). In A. sterilis, besides the two Avena characteristic bands and the hexaploid-specific band, a fourth band with $\sim 800 \mathrm{bp}$ is observed similar to the one present in A. sativa landrace from Madeira Island.

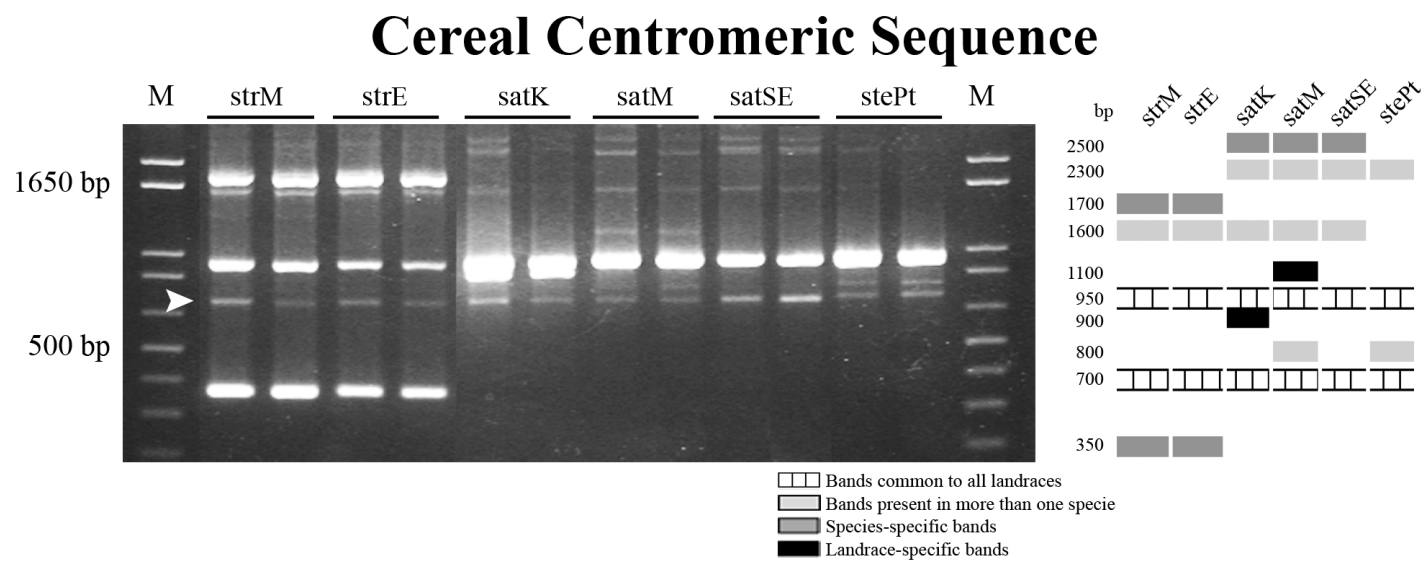

Figure 4. PCR amplification products obtained using CCS1 primers and correspondent schematic representation. M: molecular marker $1 \mathrm{~Kb}+$; strM-A. strigosa from Madeira Island; strE-A. strigosa from Elvas; satK-A. sativa "Kyto"; satM-A. sativa from Madeira Island; satSE-A. sativa "S. Eulália"; stePt-A. sterilis Pt. Arrowhead indicates the Avena characteristic band that was amplified from all genotypes, isolated and sequenced.

The Avena characteristic novel band with $~ 700 \mathrm{bp}$ was isolated, cloned and sequenced (Accession Numbers in Table S1) and the similarity values between genotypes range from $89 \%$ to $98 \%$ (Alignment 
in Figure S2). No similarity with other Avena centromeric sequences already published in Genbank was found. However, it yielded significant alignment $(\sim 72 \%)$ with retrotransposon-related centromeric sequences of Triticum (Acession Number HF541873.1) [33], Secale (Accession Number JQ963524.1) [34] and Hordeum (Accession Number AY040833.1) [35], targeting the long terminal region of the mobile element Ty3/gypsy retrotransposon Cereba firstly characterized in H. vulgare [35].

The Avena novel sequence amplified from $A$. sativa was labeled with biotin-dUTP and used as a probe for FISH evaluation in spreads of the species studied (Figure 5). In A. strigosa metaphase cells all centromeric domains are FISH-positive using that centromeric probe (Figure $5 \mathrm{a}-\mathrm{c}$ ) and although hexaploid species also revealed discrete signals, only 28 from the 42 chromosomes were FISH labeled (Figure $5 \mathrm{~d}-\mathrm{f}$ ). Thus, 14 chromosomes, probably composing one Avena haplome, do not show centromeric labeling.

Conclusively, the comparative analysis of amplification products obtained with CCS1 primers allows a clear discrimination of the three Avena species in the study. Moreover, A. sativa accessions exhibit distinct banding profiles since two landrace-specific bands were identified. The two $A$. strigosa accessions are not distinguishable, thus other molecular markers were further used to seek for a complete discrimination of all Avena genotypes analyzed.
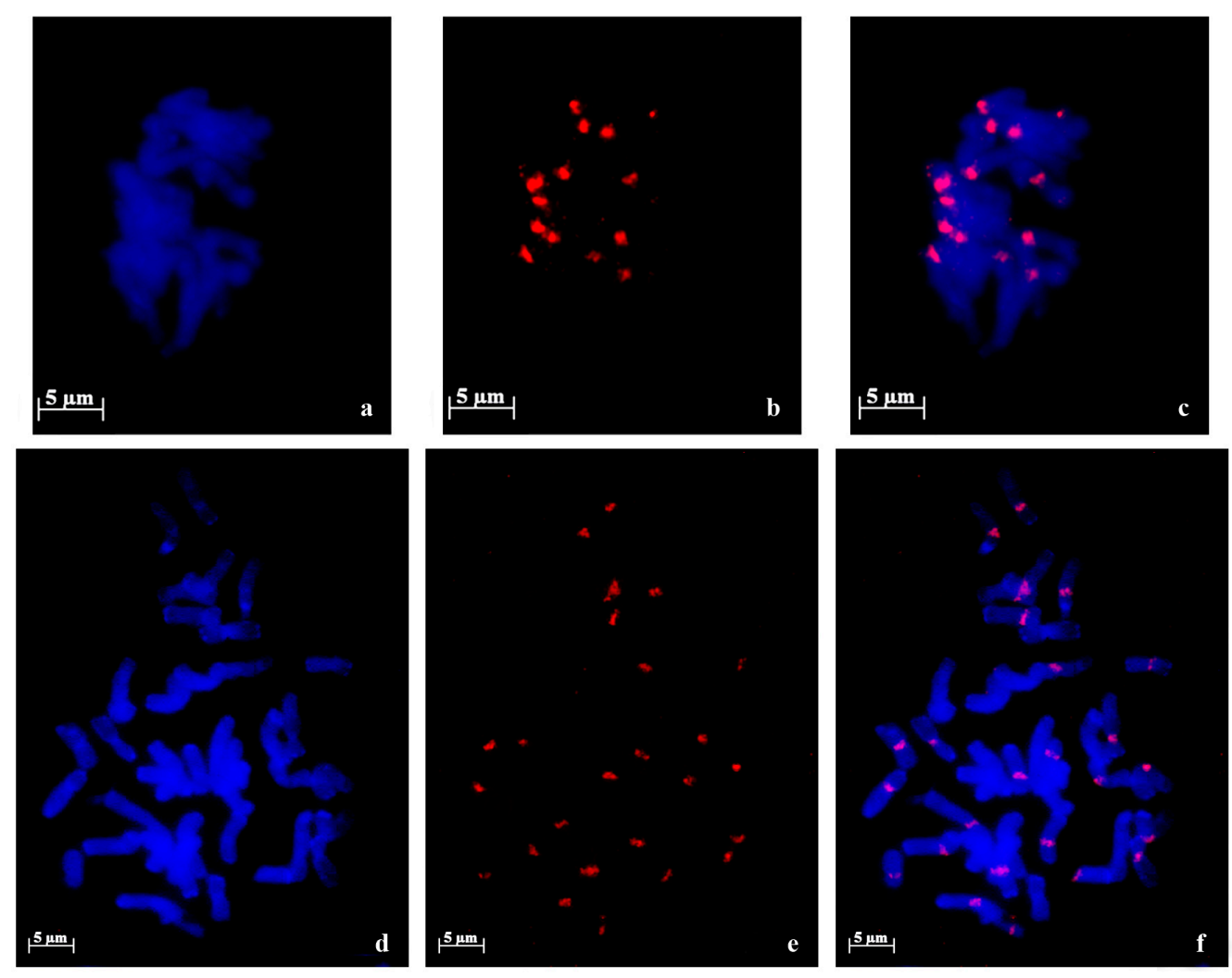

Figure 5. Centromeric sequence fluorescent in situ hybridization (red signals) in metaphase cells. Metaphase cells of A. strigosa $(\mathbf{a}-\mathbf{c})$; and A. sterilis $(\mathbf{d}-\mathbf{f})$ with DAPI staining $(\mathbf{a}, \mathbf{d})$, FISH with centromeric probe (Accession Number KM948610) (b,e) and merge of both fluorescent signals (c,f).

\subsection{Retrotransposon and Microsatellite Flanking Sequences Untangled Avena Accessions Discrimination}

The amplification of sequences flanking Nikita retrotransposon LTRs is presented in Figure 6. Banding profiles analysis allowed the identification of three bands common to all genotypes with $\sim 400$, $\sim 650$ and $\sim 1300 \mathrm{bp}$ (schematic representation in Figure 6). Both A. strigosa accessions showed identical banding patterns with two species-specific bands ( $\sim 600$ and $\sim 1700 \mathrm{bp})$ besides the three common ones. In A. sativa two landrace-specific bands were detected in "Kyto" and "S. Eulália" ( 1400 and 2400 bp, 
respectively) and $A$. sativa from Madeira Island lacks the $\sim 1250 \mathrm{bp}$ band present in all other genotypes analyzed. Finally, the $A$. sterilis Pt banding profile includes a specific band with 1100 bp (Figure 6). Thus, using the IRAP marker was not yet possible to distinguish the two A. strigosa landraces.

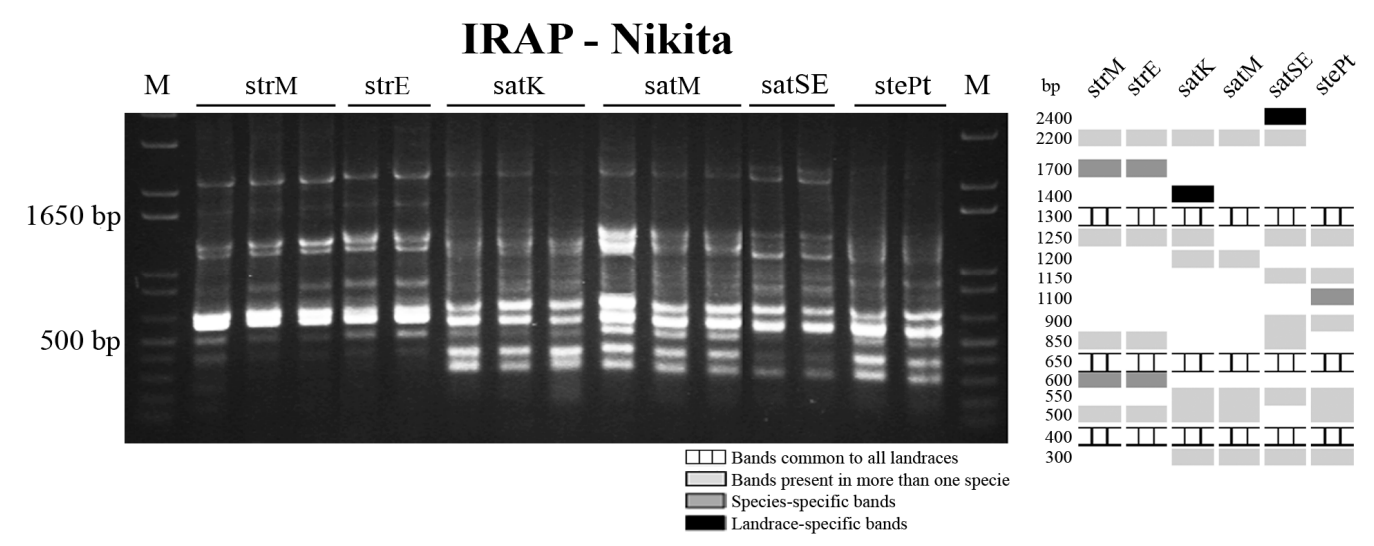

Figure 6. Inter-retrotransposon amplified polymorphism (IRAP) banding profiles obtained with primer for Nikita retrotransposon long terminal repeat (LTR) and correspondent schematic representation. M: molecular marker $1 \mathrm{~Kb}+$; strM-A. strigosa from Madeira Island; strE-A. strigosa from Elvas; satK-A. sativa "Kyto"; satM-A. sativa from Madeira Island; satSE-A. sativa "S. Eulália"; stePt-A. sterilis $\mathrm{Pt}$.

REMAP analysis performed using the same retrotransposon primer combined with the (CA)9G microsatellite anchored primer yielded a banding profile (Figure 7 with schematic representation) that includes four bands common to all Avena genotypes analyzed with $\sim 250, \sim 380, \sim 550$ and $\sim 750 \mathrm{bp}$, respectively. A. strigosa banding profile contains also four bands characteristic of this species $(\sim 280, \sim 700, \sim 950$ and $\sim 1000 \mathrm{bp})$ and the landrace from Madeira Island presents a specific one ( 200 bp). Hexaploid species profiles, besides containing the bands common to all genotypes, include two common bands (with $\sim 600$ and $\sim 1150 \mathrm{bp}$ ). Furthermore, both in $A$. sativa and A. sterilis two species-specific bands with $\sim 520$ and $\sim 780$ bp were identified. Also, two landrace-specific bands were also identified in A. sativa "Kyto" ( 100 and $~ 900$ bp) and one in "S. Eulália" ( 240 bp). Thus, the banding patterns obtained with this REMAP Nikita/(CA)9G marker allowed the clear distinction between all Avena genotypes evaluated.

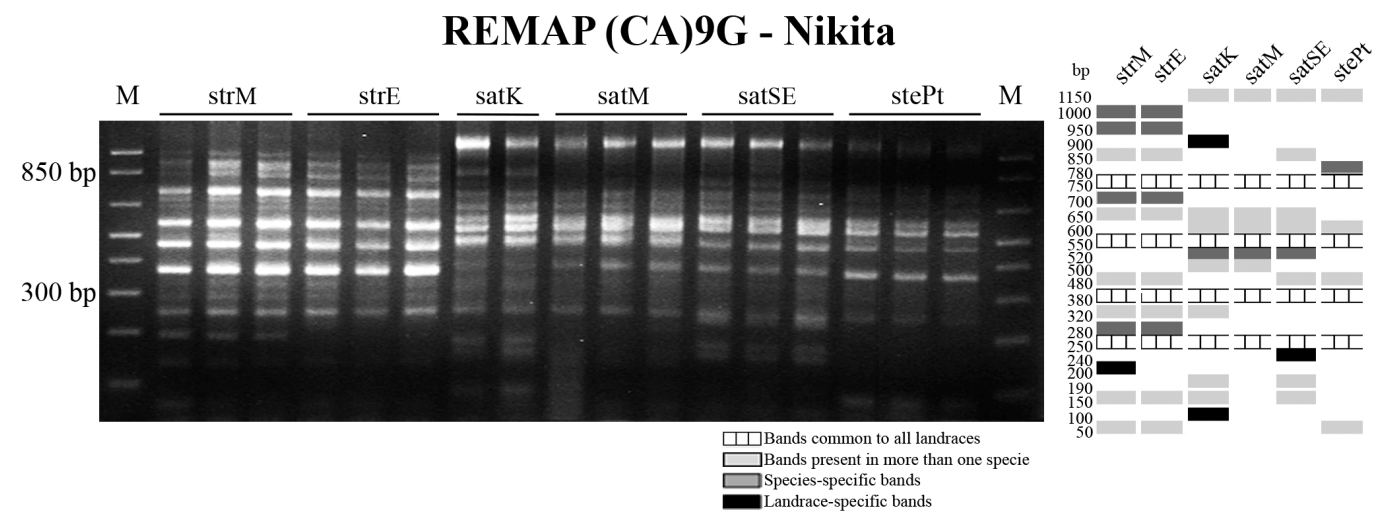

Figure 7. REMAP banding profiles obtained with primer for Nikita retrotransposon LTR and (CA)9G microsatellite anchored primer and correspondent schematic representation. M: molecular marker $1 \mathrm{~Kb}+$; strM-A. strigosa from Madeira Island; strE-A. strigosa from Elvas; satK-A. sativa "Kyto"; satM-A. sativa from Madeira Island; satSE—A. sativa "S. Eulália"; stePt—A. sterilis Pt. 
Avena species/accessions genomic diversity evaluation was performed using Cluster analysis of IRAP, REMAP and centromeric banding profiles concatenated by NTSYSpc software, and the dendrogram produced is presented in Figure 8. As expected, A. strigosa landraces revealed to be sister taxa forming a monophyletic group and the hexaploid genotypes analyzed are clustered in another group. Moreover, the three $A$. sativa genotypes evaluated are gathered in a sub-group with a similarity coefficient of $\sim 0.74$. A sativa "Kyto" and A. sativa from Madeira Island present higher similarity (with similarity coefficient $\sim 0.82$ ). Concordantly, the bootstrap value of the $A$. sativa node is the lowest one demonstrating the great similarity between the $A$. sativa genotypes analyzed. A. sterilis integrates the hexaploid Avena species branch although clearly separated from A. sativa cluster with the lowest similarity coefficient $(0.67)$.

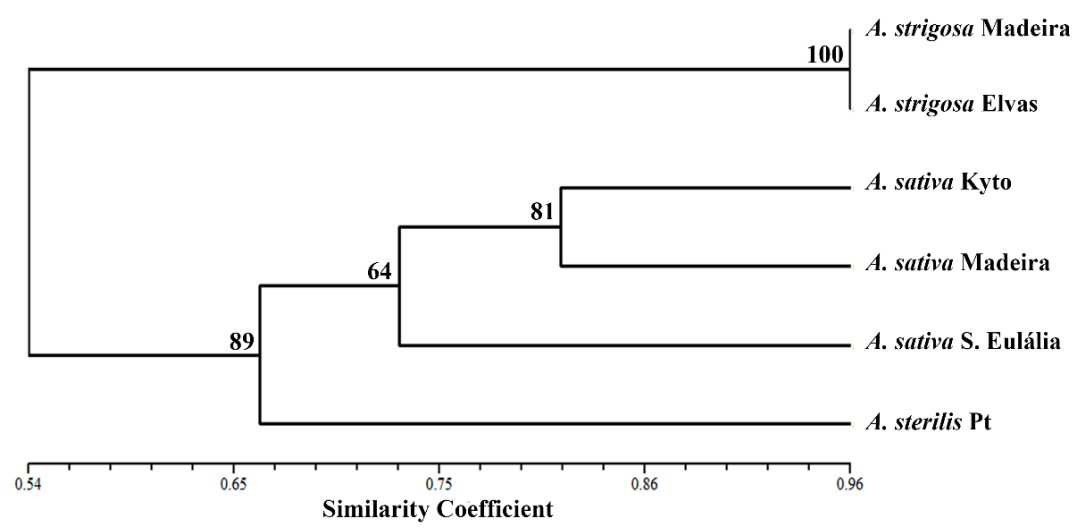

Figure 8. Dendogram based on concatenated centromeric, IRAP and REMAP banding profiles. DICE similarity index [36] and UPGMA clustering mode were used. Cophenetic correlation coefficient of 0.996 .

\section{Discussion}

In this work we aimed to portray the genomic diversity of Avena species cultivated in Portugal-A. strigosa and A. sativa, as well as the most representative Avena wild relative-A. sterilis, through molecular and cytogenetic analysis of distinct coding and non-coding repetitive sequences. The analysis of $45 \mathrm{~S}$ rDNA internal transcribed sequences revealed high similarity between $A$. strigosa, A. sativa and A. sterilis-98\% and 100\% in ITS1 and ITS2, respectively, corroborating the great homology previously reported for these sequences in several phylogenetic studies of Avena genus [8,10,11]. However, 45S rDNA assessment through fluorescent in situ hybridization (FISH) revealed some Nor loci topological diversity. In both $A$. strigosa landraces, two pairs of NORs were identified, as formerly described for other $A$. strigosa lines [37,38], although only one accession presents pairs of NORs with distinct sizes as previously described in A. strigosa accession PI 258729 [37]. In A. sativa six FISH signals were observed in accordance with the number of Nor loci previously described [37,39-42]. Though, while in "S. Eulália" all NORs exhibit similar sizes as previously reported in other A. sativa lines $[43,44]$, A. sativa landrace from Madeira Island shows NOR pairs with distinct dimensions as well as one heteromorphic Nor pair. In A. sterilis six 45S rDNA FISH signals were also detected as formerly reported for this species [37,39-41]. Moreover, distinct physical organization patterns for each pair of NORs were also described, similar to the organization inferred for A. byzantina NORs as revealed in C-banded chromosomes [42]. Thus, in contrast to the constancy of rDNA molecular analysis, Nor loci cytological evaluation allowed the identification of distinct ribosomal chromatin organization patterns.

Portuguese Avena species diversity evaluation was also studied through the analysis of other repetitive sequences previously assessed in cereals, such as pSc200 sequence-a major family of sequences isolated from rye and mapped in subtelomeric domains [32,45]. In this work we amplified in Avena a $444 \mathrm{bp}$ fragment with primers previously used in rye and wheat lines with rye chromatin 
introgression [31] and sequence analysis revealed a high level of similarity $(99 \%-100 \%)$ with rye original pSc200 sequence (Accession Number Z50039) [26]. pSc200 sequences were also detected by Southern blot and PCR as well as by FISH analysis in other Triticeae species from the genera Secale, Agropyron and Dasypyrum [26]. However, none of the Avena genotypes studied presented any detectable cytological signal, probably due to the reduced number of pSc200-like copies, substantiating the idea that the copy number of this repetitive sequences is extremely variable in distinct cereal taxa, as proposed by [26].

Contrasting with the molecular analysis of ITS and pSc200 sequences, cereal centromeric sequence (CCS1) strongly contributed to assess Avena genotypes diversity, as previously used for other cereals such as $H$. vulgare, S. cereale and T. aestivum $[27,28]$. PCR with CCS1 primers yielded consistent banding profiles that strengthen our analysis of Avena genotypes allowing the discrimination of most genotypes analyzed with the exception of the two A. strigosa landraces. CCS1 banding profiles obtained include a novel Avena band with $\sim 700$ bp with high similarity in all accessions studied. The BLAST analysis of this sequence targeted retrotransposon-related centromeric sequences isolated from cereals [33,34], showing a high similarity with the LTR of Cereba retrotransposon firstly identified in Hordeum vulgare [35], supporting therefore previous works on retrotransposon-like sequences richness in grasses centromeres [46]. FISH mapping of the CCS1 Avena-700 sequence was restricted to centromeric domains in the Avena species here studied as formerly reported for similar sequences in Triticum, Secale and Hordeum chromosomes [47]. Furthermore, our FISH analysis in diploid and hexaploid Avena species also reinforces some phylogenetic aspects in Avena genus, due to its presence in all $A$. strigosa chromosomes but only in $2 / 3$ of the chromosome complement from $A$. sativa and $A$. sterilis. This differential labeling suggests a distinct origin for $C$ genome chromosomes as already proposed by Langdon and co-workrs [46] due to evidence on the absence of conserved retrotransposon centromeric sequences from several cereal species in the $C$ genome putative progenitors of hexaploid oats such as A. eriantha and $A$. ventricosa. Moreover, the same authors also reported a reverse transcriptase probe derived from A. strigosa, the predicted A/D genome ancestor, which only labels A and D centromeric domains in A. sativa chromosomes, reinforcing therefore the suggested higher affinity of $\mathrm{A}$ and $\mathrm{D}$ genomes on hexaploid Avena (ACD) species [48].

Avena species/accessions discrimination was considerably increased by polymorphisms detected through the amplification of retrotransposons and microsatellites flanking sequences using IRAP and REMAP analysis [17]. Indeed, the accurate distinction of all Avena genotypes considered in this study was achieved through species and accession-specific bands identification, paving the way for the establishment of fast and simple molecular approaches to clearly identify Avena genotypes. Moreover, the dendogram constructed based on concatenated centromeric, IRAP and REMAP banding profiles undoubtedly allows a clear assessment of Avena diversity clustering all genotypes in two clades, one with $A$. strigosa and the other corresponding to hexaploid species, although separating A. sativa and A. sterilis accessions. Considering that REMAP technique was previously used to assess hexaploid oat species phylogenetic relationships [25] and proved to be related with oat important traits such as height [24] and cadmium accumulation [49] our work reinforces the high relevance of such molecular markers [50] also in the assessment of Avena biodiversity pool regarding both cultivated and wild relatives.

\section{Materials and Methods}

\subsection{Plant Material}

In this work we have used two Portuguese landraces of A. strigosa $(2 n=14$, AsAs); two Portuguese landraces and one line from Finland extensively used for comparative analysis of $A$. sativa $(2 n=6 x=$ AACCDD $)$; and one wild Portuguese accession of $A$. sterilis. All lines used are described in detail in Table 3. 
Seeds were germinated in petri dishes and three plants of each accession were maintained in soil pots at $16 \mathrm{~h} /$ light $\left(25^{\circ} \mathrm{C}\right)$ and $8 \mathrm{~h} /$ dark $\left(18^{\circ} \mathrm{C}\right)$. For DNA extraction fresh and healthy young leaves of 1 month-old plants were frozen and kept at $-80^{\circ} \mathrm{C}$ until further use. For cytogenetic analyses root tips were collected, cold treated for $24 \mathrm{~h}$, fixed in ethanol/acetic acid $(3: 1 v / v)$ and stored at $-20^{\circ} \mathrm{C}$ until use.

Table 3. Description of Avena accessions analyzed.

\begin{tabular}{cccc}
\hline Species & Genomic Constitution & Accession & Origin \\
\hline \multirow{2}{*}{ A. strigosa } & AsAs $2 n=14$ & $5284 /$ PRT005 & Madeira Island, Portugal \\
& & BC2531/PRT083 & Elvas, Portugal \\
\hline \multirow{2}{*}{ A. sativa } & AACCDD $2 n=6 x=42$ & "Kyto", Clav8250/USDA & Finland \\
& & "S. Eulália" /PRT083 & $\begin{array}{c}\text { Madeira, Island Portugal } \\
\text { Elvas, Portugal }\end{array}$ \\
\hline A. sterilis & AACCDD $2 n=6 x=42$ & PI267989/USDA & Portugal \\
\hline
\end{tabular}

PRT005: EAN Germplasm Bank, Oeiras; PRT083-ENMP, Elvas; USDA: United States Department of Agriculture, Agricultural Research Service.

\subsection{Genomic Analysis through PCR}

DNA extractions were performed using cetyltrimethylammonium bromide CTAB method [51]. PCR analysis was performed using different primers designed for $45 \mathrm{~S}$ rDNA internal transcribed spacers (ITS), rye subtelomeric sequence (pSc200, Accession Number Z50039) [26], cereal centromeric sequences (CCS1, Accession Number, U52217; D primer) [28], retrotransposon Nikita and microsatellites (CA)9G. Primers used are described in detail in Table 4. PCR reactions were performed in a total volume of 20 microliter with $1 \times$ PCR buffer, $1.5 \mathrm{mM} \mathrm{MgCl}_{2}, 0.25 \mathrm{mM}$ dNTP's, $1 \mathrm{mM}$ each primer, $0.5 \mathrm{U}$ Taq polymerase, $50 \mathrm{ng}$ DNA template. PCR amplification reactions of centromeric, subtelomeric and $45 \mathrm{~S}$ rDNA ITS used the following program: $5 \mathrm{~min} 94{ }^{\circ} \mathrm{C}, 30$ cycles of $45 \mathrm{~s} 94{ }^{\circ} \mathrm{C}, 45 \mathrm{~s} 60^{\circ} \mathrm{C}\left(54{ }^{\circ} \mathrm{C}\right.$ for subtelomeric sequence) and $1 \mathrm{~min}$ of $72{ }^{\circ} \mathrm{C}$, final extension of $10 \mathrm{~min}$ at $72{ }^{\circ} \mathrm{C}$. IRAP and REMAP were performed accordingly to [17]. PCR products were run on $1.7 \%$ agarose gels using as molecular weight marker $1 \mathrm{~kb}$ Plus DNA Ladder (Invitrogen, Carlsbad, CA, USA). Amplification products were detected with ethidium bromide and photographed using a Bio-Rad GEL DOC 2000 (Bio-Rad Laboratories, Inc., Hercules, CA, USA). Each PCR banding pattern was considered reproducible after being obtained in at least three technical replicates for each PCR experiment. For each sequence and each line studied at least three individual plants were analyzed. For $45 \mathrm{~S}$ rDNA internal transcribed spacers pTa71 plasmid, a $9 \mathrm{~kb}$ fragment from T. aestivum containing the 18S-5.8S-25S rDNA and intergenic spacers cloned in pUC18 [52], was also used as control template. Similarly, for pSc200 subtelomeric sequence rye genomic DNA was used as control template.

Table 4. Primers used in PCR analysis.

\begin{tabular}{cccc}
\hline Sequence & Sequence Type & Primers & Reference \\
\hline 45S ITS1 & rDNA & $\begin{array}{r}\text { Fow 5'-TCCGTAGGTGAACCTGCGG } \\
\text { Rev 5'-GCTGCGTTCTTCATCGATGC }\end{array}$ & {$[29]$} \\
\hline \multirow{2}{*}{45 ITS2 } & rDNA & $\begin{array}{r}\text { Fow 5'-GCATCGATGAAGAACGCAGC } \\
\text { Rev 5'-TCCTCCGCTTATTGATATGC }\end{array}$ & {$[29]$} \\
\hline pSc200 & Telomeric & $\begin{array}{r}\text { Fow 5'-TCTTTGATCACCGTTTCTTCG } \\
\text { Rev 5'-CCCCACCCATGTATGGATAA }\end{array}$ & {$[31]$} \\
\hline CCS1 & Centromeric & 5'-GGTGCCCGATCTTTCGATGAGA & {$[28]$} \\
\hline Nikita LTR & Retrotransposon & $5^{\prime}$-CGCTCCAGCGGTACTGCC & {$[53]$} \\
\hline CA 9 GG & Microsatellite & $5^{\prime}$-CACACACACACACACACAG & - \\
\hline
\end{tabular}


Selected bands were gel isolated, purified using High Pure PCR Product Purification Kit (Roche, Basel, Switzerland), cloned using TOPO TA Cloning Kit in pCR2.1 vector (Invitrogen, Carlsbad, CA, USA) and sequenced. At least three distinct clone per sequence/line were analyzed.

\subsection{Sequence Similarity Analysis}

Sequences from isolated bands were verified and aligned using BioEdit version 7.1.3.0 [54] and analyzed along with sequences obtained from DDBJ/EMBL/GenBank International Nucleotide Sequence Database. For sequence similarity analyses Clustal W2 [30] was used.

Inter Retrotransposons Amplified Polymorphism (IRAP), Retrotransposons Microsatellite Amplified Polymorphism (REMAP) and centromeric sequences PCR banding profiles obtained were transformed in binary matrices, where 1 corresponds to band presence and 0 to absence. NTSYSpc software [55] was used to infer genetic similarity between all accessions and species using Dice coefficient [36]. Dendrograms were obtained through UPGMA (Unweighted Pair Group Method with Arithmetic Mean) method for a concatenated binary of CCS1, IRAP and REMAP profiles obtained using Concatenator Software [56]. Cophenetic correlation coefficient $(r)$ was estimated to verify the adjustment between similarity matrix and respective dendrogram-derived matrix. The determination of confidence limits by bootstrap analysis was done using the WINBOOT software.

\subsection{Cytogenetics Analysis through Fluorescent in Situ Hybridization (FISH)}

FISH analysis was performed as described in [45] in meristematic cells spreads obtained from root tips fixed in ethanol/acetic acid (3:1) and digested with pectinase/cellulose. Pre-hybridization treatments to remove proteins and RNA were performed with $6.67 \mu \mathrm{g} / \mathrm{mL}$ pepsin and $100 \mathrm{ng} / \mu \mathrm{L}$ RNAse, respectively. pTa71 probe, a plasmid that includes a $9 \mathrm{~kb}$ EcoRI fragment of the rDNA unit from wheat (Triticum aestivum) [52] was labeled with digoxigenin-dUTP or biotin-dUTP (Roche, Basel, Switzerland) through Nick Translation. Centromeric sequence (Accession Number KM948610) was amplified from A. sativa and labeled with biotin-dUTP (Roche) through PCR using CCS1 primers. The subtelomeric probe pSc200 was amplified from A. sativa DNA and labeled with digoxigenin-dUTP through PCR. For each slide $50 \mathrm{ng}$ of pTa71, $100 \mathrm{ng}$ of centromeric sequence and $100 \mathrm{ng}$ of pSc200 were diluted in a hybridization mixture composed by 50\% formamide, $2 \times$ SSC (saline-sodium citrate buffer), $0.17 \%$ SDS (sodium dodecyl sulfate), $1 \mu \mathrm{g} / \mu \mathrm{L}$ salmon sperm, $10 \%$ dextrane sulphate (stringency of $77 \%$ ). Post-hybridization washes were done with a stringency of $85 \%$ and bovine serum albumin $5 \%(w / v)$ was used as blocking reagent before probe detection with $2 \mu \mathrm{g} / \mathrm{mL}$ of Anti-digoxigenin Fluorescein (FITC) and $5 \mu \mathrm{g} / \mathrm{mL}$ Streptavidin-Cy3. Cells were counterstained with 4',6-diamidino-2-phenylindole hydrochloride (DAPI) in Citifluor antifade mounting medium (AF1; Agar Scientific, Stansted, Essex, UK). Samples were examined using a Zeiss AxioImager Z1 epifluorescence microscope. Images were obtained using a Zeiss AxioCam HRm digital camera (Zeiss, Oberkochen, Germany) and the selected ones were processed using Photoshop (Adobe Systems, San Jose, CA, USA). The described FISH signals illustrated in Figures 1, 2 and 5 were observed in at least 10 metaphase or prometaphase cells. $45 \mathrm{~S}$ rDNA labeling was analyzed through the comparison of FISH signals sizes within each cell, considering that all chromosomes in the same cell assume a similar condensation level.

\section{Conclusions}

Genomic diversity and molecular discrimination of the most relevant crop and weed Avena species from Portugal were assessed through molecular and cytological evaluation of distinct repetitive sequences. FISH analysis of $45 \mathrm{~S}$ rDNA revealed inter and intra-specific variability though the ITS high similarity observed. pSc200-like sequences were identified and revealed high homology in the distinct genotypes analyzed. Conversely, the analysis of centromeric banding patterns along with profiles produced by retrotransposons and microsatellites targeting methodologies (IRAP and REMAP) 
allowed an accurate assessment of the variability, providing novel prospective molecular markers for future oat breeding programs.

Supplementary Materials: Supplementary materials can be found at http://www.mdpi.com/1422-0067/ $17 / 2 / 203 /$ s1.

Acknowledgments: We would like to thank Augusta Barão for her excellent support to obtain fluorescent in situ images. Diana Tomás was funded by a Fundação para a Ciência e a Tecnologia, Portugal (FCT) doctoral scholarship (SFRH/BD/93156/2013), Manuela Silva by the FCT Investigator Programme (IF/00834/2014) and the research work was financed by FCT project PTDC/BIA-BEC/101964/2008, and FCT LEAF (Linking Landscape, Environment, Agriculture and Food) Unit (UID/AGR/04129/2013).

Author Contributions: Experiments conception and design: Diana Tomás, Wanda Viegas, Manuela Silva. Experiments performance: Diana Tomás, Joana Rodrigues, Ana Varela. Data analysis: Diana Tomás, Joana Rodrigues, Manuela Silva, Wanda Viegas. Plant materials contribution: Maria Manuela Veloso. Paper writing: Diana Tomás, Wanda Viegas, Manuela Silva.

Conflicts of Interest: The authors declare no conflict of interest.

\section{References}

1. Loskutov, I.G. On evolutionary pathways of Avena species. Genet. Resour. Crop. Evol. 2008, 55, 211-220. [CrossRef]

2. Baum, B.R. Oats: Wild and Cultivated: A Monograph of the Genus Avena L. (Poaceae); Biosystematics Research Institute, Canada Department of Agriculture, Research Branch: Ottawa, AB, Canada, 1977.

3. Costa, J.C.; Balsinhas, A.; Cabral, F.; Moreira, I. Distribuição e diferenciação isoenzimática de táxones do género Avena L. Portugaliae Acta Biol. 2000, 19, 373-386.

4. Loskutov, I.G. Interspecific crosses in the genus Avena L. Russ. J. Genet. 2001, 37, 467-475. [CrossRef]

5. Loskutov, I.; Rines, H. Avena . In Wild Crop Relatives: Genomic and Breeding Resources; Kole, C., Ed.; Springer: Berlin/Heidelberg, Germany, 2011; pp. 109-183.

6. Fu, Y.B.; Williams, D.J. AFLP variation in 25 Avena species. Theor. Appl. Genet. 2008, 117, 333-342. [CrossRef] [PubMed]

7. Li, W.T.; Peng, Y.Y.; Wei, Y.M.; Baum, B.R.; Zheng, Y.L. Relationships among Avena species as revealed by consensus chloroplast simple sequence repeat (ccSSR) markers. Genet. Resour. Crop. Evol. 2009, 56, 465-480. [CrossRef]

8. Rodionov, A.V.; Tyupa, N.B.; Kim, E.S.; Machs, E.M.; Loskutov, I.G. Genomic configuration of the autotetraploid oat species Avena macrostachya inferred from comparative analysis of ITS1 and ITS2 sequences: On the oat karyotype evolution during the early events of the Avena species divergence. Russ. J. Genet. 2005, 41, 518-528. [CrossRef]

9. Nikoloudakis, N.; Katsiotis, A. The origin of the C-genome and cytoplasm of Avena polyploids. Theor. Appl. Genet. 2008, 117, 273-281. [CrossRef] [PubMed]

10. Nikoloudakis, N.; Skaracis, G.; Katsiotis, A. Evolutionary insights inferred by molecular analysis of the ITS1-5.8S-ITS2 and IGS Avena sp sequences. Mol. Phylogenet. Evol. 2008, 46, 102-115. [CrossRef] [PubMed]

11. Peng, Y.Y.; Baum, B.R.; Ren, C.Z.; Jiang, Q.T.; Chen, G.Y.; Zheng, Y.L.; Wei, Y.M. The evolution pattern of rDNA ITS in Avena and phylogenetic relationship of the Avena species (Poaceae: Aveneae). Hereditas 2010, 147, 183-204. [CrossRef] [PubMed]

12. Da-Silva, P.R.; Milach, S.C.; Tisian, L.M. Transferability and utility of white oat (Avena sativa) microsatellite markers for genetic studies in black oat (Avena strigosa). Genet. Mol. Res. 2011, 10, 2916-2923. [CrossRef] [PubMed]

13. Boczkowska, M.; Tarczyk, E. Genetic diversity among polish landraces of common oat (Avena sativa L.). Genet. Resour. Crop Evol. 2013, 60, 2157-2169. [CrossRef]

14. Montilla-Bason, G.; Sanchez-Martin, J.; Rispail, N.; Rubiales, D.; Mur, L.; Langdon, T.; Griffiths, I.; Howarth, C.; Prats, E. Genetic diversity and population structure among oat cultivars and landraces. Plant Mol. Biol. Rep. 2013, 31, 1305-1314. [CrossRef]

15. Fu, Y.B.; Chong, J.; Fetch, T.; Wang, M.L. Microsatellite variation in Avena sterilis oat germplasm. Theor. Appl. Genet. 2007, 114, 1029-1038. [CrossRef] [PubMed] 
16. Goffreda, J.C.; Burnquist, W.B.; Beer, S.C.; Tanksley, S.D.; Sorrells, M.E. Application of molecular markers to assess genetic-relationships among accessions of wild oat, Avena sterilis. Theor. Appl. Genet. 1992, 85, 146-151. [CrossRef] [PubMed]

17. Kalendar, R.; Grob, T.; Regina, M.; Suoniemi, A.; Schulman, A. IRAP and REMAP: Two new retrotransposon-based DNA fingerprinting techniques. Theor. Appl. Genet. 1999, 98, 704-711. [CrossRef]

18. Vitte, C.; Panaud, O. LTR retrotransposons and flowering plant genome size: Emergence of the increase/decrease model. Cytogenet. Genome Res. 2005, 110, 91-107. [CrossRef] [PubMed]

19. Ramsay, L.; Macaulay, M.; Cardle, L.; Morgante, M.; degli Ivanissevich, S.; Maestri, E.; Powell, W.; Waugh, R. Intimate association of microsatellite repeats with retrotransposons and other dispersed repetitive elements in barley. Plant J. 1999, 17, 415-425. [CrossRef] [PubMed]

20. Bento, M.; Gustafson, J.P.; Viegas, W.; Silva, M. Size matters in Triticeae polyploids: Larger genomes have higher remodeling. Genome 2011, 54, 175-183. [PubMed]

21. Branco, C.J.; Vieira, E.A.; Malone, G.; Kopp, M.M.; Malone, E.; Bernardes, A.; Mistura, C.C.; Carvalho, F.I.; Oliveira, C.A. IRAP and REMAP assessments of genetic similarity in rice. J. Appl. Genet. 2007, 48, 107-113. [CrossRef] [PubMed]

22. Kuhn, B.C.; Lopez-Ribera, I.; Machado, M.D.P.D.; Vicient, C.M. Genetic diversity of maize germplasm assessed by retrotransposon-based markers. Electrophoresis 2014, 35, 1921-1927. [CrossRef] [PubMed]

23. Doungous, O.; Kalendar, R.; Adiobo, A.; Schulman, A. Retrotransposon molecular markers resolve cocoyam (Xanthosoma sagittifolium) and taro (Colocasia esculenta) by type and variety. Euphytica 2015, 206, 1-14. [CrossRef]

24. Tanhuanpaa, P.; Kalendar, R.; Laurila, J.; Schulman, A.H.; Manninen, O.; Kiviharju, E. Generation of SNP markers for short straw in oat (Avena sativa L.). Genome 2006, 49, 282-287. [CrossRef] [PubMed]

25. Paczos-Grzeda, E.; Bednarek, P.T. Comparative analysis of hexaploid Avena species using REMAP and ISSR methods. Turk. J. Bot. 2014, 38, 1103-1111. [CrossRef]

26. Vershinin, A.V.; Alkhimova, E.G.; HeslopHarrison, J.S. Molecular diversification of tandemly organized DNA sequences and heterochromatic chromosome regions in some triticeae species. Chromosome Res. 1996, 4, 517-525. [CrossRef] [PubMed]

27. Abbo, S.; Dunford, R.P.; Foote, T.N.; Reader, S.M.; Flavell, R.B.; Moore, G. Organization of retro-element and stem-loop repeat families in the genomes and nuclei of cereals. Chromosome Res. 1995, 3, 5-15. [CrossRef] [PubMed]

28. Aragon-Alcaide, L.; Miller, T.; Schwarzacher, T.; Reader, S.; Moore, G. A cereal centromeric sequence. Chromosoma 1996, 105, 261-268. [CrossRef] [PubMed]

29. White, T.; Bruns, T.; Lee, S.; Taylor, J. Amplification and direct sequencing of fungal ribosomal RNA genes for phylogenetics. In PCR Protocols: A Guide to Methods and Applications; Innis, M., Gelfand, D., Shinsky, J., White, T., Eds.; Academic Press: New York, NY, USA, 1990; pp. 315-322.

30. Larkin, M.A.; Blackshields, G.; Brown, N.P.; Chenna, R.; McGettigan, P.A.; McWilliam, H.; Valentin, F.; Wallace, I.M.; Wilm, A.; Lopez, R.; et al. Clustal W and clustal X version 2.0. Bioinformatics 2007, 23, 2947-2948. [CrossRef] [PubMed]

31. Bento, M.; Gustafson, P.; Viegas, W.; Silva, M. Genome merger: From sequence rearrangements in triticale to their elimination in wheat-rye addition lines. Theor. Appl. Genet. 2010, 121, 489-497. [CrossRef] [PubMed]

32. Vershinin, A.V.; Schwarzacher, T.; Heslopharrison, J.S. The large-scale genomic organization of repetitive DNA families at the telomeres of rye chromosomes. Plant Cell 1995, 7, 1823-1833. [CrossRef] [PubMed]

33. Li, B.C.; Choulet, F.; Heng, Y.F.; Hao, W.W.; Paux, E.; Liu, Z.; Yue, W.; Jin, W.W.; Feuillet, C.; Zhang, X.Y. Wheat centromeric retrotransposons: The new ones take a major role in centromeric structure. Plant J. 2013, 73, 952-965. [CrossRef] [PubMed]

34. Banaei-Moghaddam, A.M.; Schubert, V.; Kumke, K.; Weiss, O.; Klemme, S.; Nagaki, K.; Macas, J.; Gonzalez-Sanchez, M.; Heredia, V.; Gomez-Revilla, D.; et al. Nondisjunction in favor of a chromosome: The mechanism of rye B chromosome drive during pollen mitosis. Plant Cell 2012, 24, 4124-4134. [CrossRef] [PubMed]

35. Hudakova, S.; Michalek, W.; Presting, G.G.; ten Hoopen, R.; dos Santos, K.; Jasencakova, Z.; Schubert, I. Sequence organization of barley centromeres. Nucleic Acids Res. 2001, 29, 5029-5035. [CrossRef] [PubMed]

36. Dice, L.R. Measures of the amount of ecologic association between species. Ecology 1945, 26, $297-302$. [CrossRef] 
37. Linares, C.; Gonzalez, J.; Ferrer, E.; Fominaya, A. The use of double fluorescence in situ hybridization to physically map the positions of 5S rDNA genes in relation to the chromosomal location of 18S-5.8S-26S rDNA and a C genome specific DNA sequence in the genus Avena. Genome 1996, 39, 535-542. [CrossRef] [PubMed]

38. Badaeva, E.D.; Shelukhina, O.Y.; Goryunova, S.V.; Loskutov, I.G.; Pukhalskiy, V.A. Phylogenetic relationships of tetraploid AB-genome Avena species evaluated by means of cytogenetic (C-banding and FISH) and RADP analyses. J. Bot. 2010, 2010. [CrossRef]

39. Badaeva, E.D.; Shelukhina, O.Y.; Dedkova, O.S.; Loskutov, I.G.; Pukhalskyi, V.A. Comparative cytogenetic analysis of hexaploid Avena L. Species. Russ. J. Genet. 2011, 47, 691-702. [CrossRef]

40. Fominaya, A.; Hueros, G.; Loarce, Y.; Ferrer, E. Chromosomal distribution of a repeated DNA sequence from $\mathrm{C}$-genome heterochromatin and the identification of a new ribosomal DNA locus in the Avena genus. Genome 1995, 38, 548-557. [CrossRef] [PubMed]

41. Jellen, E.N.; Phillips, R.L.; Rines, H.W. C-banded karyotypes and polymorphisms in hexaploid oat accessions (Avena spp.) using Wright's stain. Genome 1993, 36, 1129-1137. [CrossRef] [PubMed]

42. Linares, C.; Vega, C.; Ferrer, E.; Fominaya, A. Identification of C-banded chromosomes in meiosis and the analysis of nucleolar activity in Avena byzantina C. Koch cv "Kanota". TAG. Theor. Appl. Genet. 1992, 83, 650-654. [CrossRef] [PubMed]

43. Irigoyen, M.L.; Linares, C.; Ferrer, E.; Fominaya, A. Fluorescence in situ hybridization mapping of Avena sativa L. cv. Sunii and its monosomic lines using cloned repetitive DNA sequences. Genome 2002, 45, 1230-1237. [CrossRef] [PubMed]

44. Linares, C.; Ferrer, E.; Fominaya, A. Discrimination of the closely related a and d genomes of the hexaploid oat Avena sativa L. Proc. Natl. Acad. Sci. USA 1998, 95, 12450-12455. [CrossRef] [PubMed]

45. Tomas, D.; Brazao, J.; Viegas, W.; Silva, M. Differential effects of high-temperature stress on nuclear topology and transcription of repetitive noncoding and coding rye sequences. Cytogenet. Genome Res. 2013, 139, 119-127. [CrossRef] [PubMed]

46. Langdon, T.; Seago, C.; Mende, M.; Leggett, M.; Thomas, H.; Foster, J.W.; Thomas, H.; Jones, R.N.; Jenkins, G. Retrotransposon evolution in diverse plant genomes. Genetics 2000, 156, 313-325. [PubMed]

47. Presting, G.G.; Malysheva, L.; Fuchs, J.; Schubert, I. A TY3/GYPSY retrotransposon-like sequence localizes to the centromeric regions of cereal chromosomes. Plant J. 1998, 16, 721-728. [CrossRef] [PubMed]

48. Fujigaki, J.; Tsuchiya, T. Karyotype analysis in a haploid plant of an inbred rye, Secale cereale 1, by acetocarmine-giemsa staining technique. Z. Pflanzenzucht 1985, 94, 234-243.

49. Tanhuanpaa, P.; Kalendar, R.; Schulman, A.H.; Kiviharju, E. A major gene for grain cadmium accumulation in oat (Avena sativa L.). Genome 2007, 50, 588-594. [PubMed]

50. Kalendar, R.; Schulman, A. Transposon-based tagging: IRAP, REMAP, and IPBS. In Molecular Plant Taxonomy; Besse, P., Ed.; Humana Press: Totowa, NJ, USA, 2014; Volume 1115, pp. 233-255.

51. Doyle, J.J.; Doyle, J.L. A rapid DNA isolation procedure for small quantities of fresh leaf tissue. Phytochem. Bull. 1987, 19, 11-15.

52. Gerlach, W.L.; Bedbrook, J.R. Cloning and characterization of ribosomal-RNA genes from wheat and barley. Nucleic Acids Res. 1979, 7, 1869-1885. [CrossRef] [PubMed]

53. Baumel, A.; Ainouche, M.; Kalendar, R.; Schulman, A.H. Retrotransposons and genomic stability in populations of the young allopolyploid species Spartina anglica C.E. Hubbard (Poaceae). Mol. Biol. Evol. 2002, 19, 1218-1227. [CrossRef] [PubMed]

54. Hall, T.A. Bioedit: A user-friendly biological sequence alignment editor and analysis program for windows 95/98/nt. Nucleic Acids Symp. Ser. 1999, 41, 95-98.

55. Rohlf, F.J. NTSYS-pc: Numerical Taxonomy and Multivariate Analysis System, Version 2.1; Applied Biostatistics Inc.: New York, NY, USA, 2000.

56. Pina-Martins, F.; Paulo, O.S. Concatenator: Sequence data matrices handling made easy. Mol. Ecol. Resour. 2008, 8, 1254-1255. [CrossRef] [PubMed]

(c) 2016 by the authors; licensee MDPI, Basel, Switzerland. This article is an open access article distributed under the terms and conditions of the Creative Commons by Attribution (CC-BY) license (http:/ / creativecommons.org/licenses/by/4.0/). 